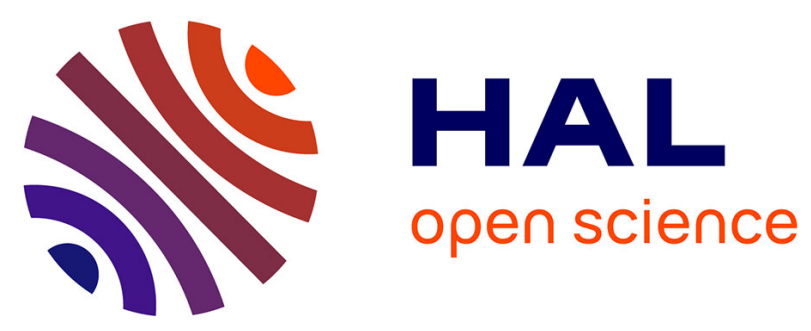

\title{
Towards a Spatial Ability Training to Improve Mental Imagery based Brain-Computer Interface (MI-BCI) Performance: a Pilot Study
}

\author{
Suzy Teillet, Fabien Lotte, Bernard N'Kaoua, Camille Jeunet
}

\section{To cite this version:}

Suzy Teillet, Fabien Lotte, Bernard N'Kaoua, Camille Jeunet. Towards a Spatial Ability Training to Improve Mental Imagery based Brain-Computer Interface (MI-BCI) Performance: a Pilot Study. IEEE International Conference on Systems, Man, and Cybernetics (SMC 2016), Oct 2016, Budapest, Hungary. pp.6. hal-01341042

\section{HAL Id: hal-01341042 \\ https://hal.inria.fr/hal-01341042}

Submitted on 9 Jul 2016

HAL is a multi-disciplinary open access archive for the deposit and dissemination of scientific research documents, whether they are published or not. The documents may come from teaching and research institutions in France or abroad, or from public or private research centers.
L'archive ouverte pluridisciplinaire HAL, est destinée au dépôt et à la diffusion de documents scientifiques de niveau recherche, publiés ou non, émanant des établissements d'enseignement et de recherche français ou étrangers, des laboratoires publics ou privés. 


\section{Towards a Spatial Ability Training to Improve Mental Imagery based Brain-Computer Interface (MI-BCI) Performance: a Pilot Study}

\author{
Suzy TEILLET \\ Inria Bordeaux Sud-Ouest \\ Talence, France \\ suzy.teillet@inria.fr
}

\author{
Fabien LOTTE \\ Inria Bordeaux Sud-Ouest/LaBRI \\ Talence, France \\ fabien.lotte@inria.fr
}

\author{
Bernard N'Kaoua \\ University of Bordeaux \\ Bordeaux, France \\ bernard.nkaoua@u-bordeaux.fr
}

\author{
Camille JEUNET $*$ \\ University of Bordeaux/Inria \\ Bordeaux, France \\ camille.jeunet@inria.fr
}

\begin{abstract}
Although Mental Imagery based Brain-Computer Interfaces (MI-BCIs) seem to be very promising for many applications, they are still rarely used outside laboratories. This is partly due to suboptimal training protocols, which provide little help to users learning how to control the system. Indeed, they do not take into account recommendations from instructional design. However, it has been shown that MI-BCI performances are significantly correlated to certain aspects of the users' cognitive profile, such as their Spatial Abilities (SA). Thus, it remains to be elucidated whether training the SA of BCI users would also improve their BCI control performance. Therefore, we proposed and validated an $\mathrm{SA}$ training that aimed at being included in an MI-BCI training protocol. Our pre-studies indeed confirmed that such a training does increase people's SA abilities. We then conducted a pilot study with 3 participants, one with a standard MI-BCI training protocol, one with the proposed SA training integrated into a standard MI-BCI training, and another control integrating another training, here verbal comprehension tasks, into a standard MI-BCI training. While such a small population cannot lead to any strong result, our first results show that SA training can indeed be integrated into MI-BCI training and is thus worth being further investigated for BCI user training.

Index Terms-Brain-Computer Interfaces, Training, Spatial Abilities, Mental Rotation
\end{abstract}

\section{INTRODUCTION}

Brain-computer interfaces (BCIs) are communication and control systems enabling users to interact with their environment using their brain activity alone [1] which is often measured using Electroencephalography (EEG). A prominent type of BCI, called Mental-Imagery based BCI (MI-BCI), makes use of control signals sent via the execution of mentalimagery tasks, such as imagining movements of the left hand vs. right hand. Such technologies are very promising, notably in the context of stroke rehabilitation [2]. However, MI-BCIs remain barely used outside laboratories due to their lack of reliability [1]. Two main factors responsible for this low reliability have been identified. The first, extensively investigated, concerns brain signal processing with current classification algorithms being still imperfect [3]. The second concerns the users themselves: between $15 \%$ and $30 \%$ cannot control a BCI at all (so-called "BCI deficiency"), while most of the remaining $80 \%$ obtain relatively modest performances [3].

978-1-4799-8697-2/15/\$31.00 (C)2015 European Union
It is now accepted that controlling an MI-BCI requires the acquisition of specific skills, and particularly the ability to generate stable and distinct brain activity patterns while performing the different MI-tasks [4], [5]. Just as with any skill, appropriate training is required to acquire these skills [4]. Yet, current standard training protocols, which do not take into account the recommendations from psychology and instructional design (such as offering adaptive and progressive tasks or explanatory, supportive and multimodal feedback), appear to be theoretically inappropriate, and thus might be partly responsible for BCI illiteracy and modest user performance [6].

In a previous study, we showed that the user's profile could be related to MI-BCI control abilities based on a 6-session protocol (i.e., over 6 different days) [7]. In this experiment, the participants $(\mathrm{N}=18)$ had to learn to perform 3 MI tasks: left-hand motor imagery, mental rotation and mental calculation. The results stressed the correlation between mental rotation scores (measured using questionnaires, [8]) which reflect Spatial Abilities (SA), and mean MI-BCI performance $[\mathrm{r}=0.696, \mathrm{p} \leq 0.05]$. SA are the mental capacities which enable the construction, transformation and interpretation of mental images. Based on these results, it seems that users with high mental rotation scores perform better when using an MI-BCI than users with low mental rotation scores. Recently, a second study [9], involving 20 healthy participants training to control a 2-class MI-BCI (left- and right-hand movement imagination), revealed a similar correlation between peak MI-BCI performance and mental rotation scores [ $\mathrm{r}=0.464, \mathrm{p} \leq 0.05]$, thus reinforcing the hypothesis of a close relationship between spatial abilities and MI-BCI control performance.

With a view to improving users' MI-BCI control abilities, further investigating this relationship between MI-BCI performance and SA seems promising. More specifically, beyond the correlation, it would be interesting to assess whether a causal relationship exists between SA and MI-BCI performance. In other words, does an improvement in SA lead to improved MIBCI performance? This raised the idea of a new approach for MI-BCI training by targeting the improvement of users' SA. Therefore, we implemented an SA training (composed of 6 sessions: 1 standard MI-BCI session - 3 sessions of SA training 
- 2 standard MI-BCI sessions) with the long term aim of testing its efficiency in terms of MI-BCI performance improvement. Here, we validate this SA training protocol together (1) with a standard MI-BCI training protocol (6 MI-BCI sessions) and (2) with a similarly structured verbal comprehension training protocol (i.e., composed of 6 sessions: 1 standard MI-BCI session - 3 sessions of verbal comprehension training - 2 standard MI-BCI sessions), as control trainings. We chose to train verbal comprehension because it would appear that, based on the literature, this skill is independent from SA skills. In this way, the verbal comprehension training should not have any impact on users' SA, but will enable us to control that any improvement in MI-BCI performance will be due to the SA training, and not just to a different cognitive training.

Hereafter we will first explain the design of the three training protocols used, namely the Mental Imagery (MI), Spatial Ability (SA) and Verbal Comprehension (VC) training protocols. In the second and third parts, we will describe the two pre-studies carried out, the goal of which was on the one hand to characterise the complexity of the different exercises proposed in the SA and VC trainings, and on the other hand to validate the SA training (i.e., to check that it does actually enable the participants to improve their SA). Finally, we will describe the preliminary results of the validation, with actual BCI use, of the standard MI-BCI protocol, the SA and the VC training protocols.

\section{Design of our Mental Imagery (Mi), Spatial ABILITY (SA) AND VERBAL COMPREHENSION (VC) TRAINING PROTOCOLS}

In this section, we will describe the principle of each training protocol. We wanted the SA and VC training sessions to be comparable to a standard MI-BCI training session in terms of training duration and structure. Thus, similarly to a standard MI-BCI training session, all training sessions (MI, $\mathrm{SA}$ and VC) were composed of 5 runs of around 7 minutes each, and therefore lasted around $40 \mathrm{~min}$ in total. Among the 5 runs, the first one was a calibration or training run (during which no feedback was provided) and the 4 subsequent runs were test runs (with feedback indicating success at the task).

\section{A. Mental Imagery training sessions}

The goal of these training sessions is to learn to perform three different Mental-Imagery (MI) tasks, namely, a left-hand motor-imagery task, a mental rotation task and a mental subtraction task. These tasks were chosen based on the results of [10], which suggest that the combination of these tasks was associated with the best performance on average across subjects. "Left-hand motor imagery" (LHAND) refers to the continuous kinesthetic imagination of a left-hand movement, chosen by the participant, without any actual movement. "Mental rotation" (ROTATION) and "mental subtraction" (SUBTRACTION) correspond respectively to the mental visualisation of a 3 Dimensional shape rotating in a 3 Dimensional space and to successive subtractions of a 3-digit number by a 2-digit number (ranging between 11 and 19), both being randomly generated and displayed on a screen. As stated earlier, an MI training session was composed of 5 runs of around $7 \mathrm{~min}$ each. During each run, participants had to perform 45 trials (15 trials per task x 3 MI-tasks, presented in a random order), with each trial lasting 8 s. Figure 1 represents a testing trial, i.e., a trial with feedback. The first run of each session had a similar structure but no feedback was provided during the last $4 \mathrm{~s}$. For more details concerning this training protocol, please refer to our previous study [7].

\section{B. Spatial Ability training sessions}

The objective of the Spatial Ability (SA) training was to improve this skill by performing different kinds of mental rotation exercises. Based on the recommendations from instructional design [11], which have shown that variability in training tasks leads to better learning, we proposed different kinds of exercises, 4 in total (see Figure 2), theoretically associated with a different degree of difficulty. Indeed, two exercises comprised 2D rotations while the other two were associated with 3D rotations. During each session, participants had to perform 5 runs, each of them lasting 7 minutes. At each run a different exercise was presented, but the instructions were always the same: a target figure was displayed at the top of the screen, followed by a further four figures below ; among these four figures, two corresponded to the target figure that had been rotated and two were mirror images of the target figure. The participant had to select the two correct proposals, i.e. the two rotated figures. A time limit of 7 minutes was set, during which participants had to answer as many questions as possible. From the second run onwards, participants were able to click on a check button in order to receive feedback (i.e., to know whether they had answered correctly or not).

\section{Verbal Comprehension training sessions}

Verbal Comprehension (VC) training was used as a control condition. Indeed, to our knowledge, VC skills are neither related to SA nor to MI-BCI performance. The objective of the $\mathrm{VC}$ training was to improve this skill by performing different

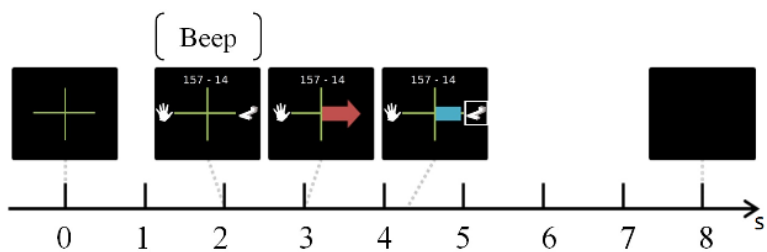

Fig. 1. Example of a trial where the participant had to perform mental rotation. At $\mathrm{t}=0 \mathrm{~s}$, a fixation cross appears on the screen. The participant has to focus on the cross to avoid eye movements. Then, at $t=2 \mathrm{~s}$, a beep announces the incoming instruction and pictograms representing the tasks are displayed (the hand on the left represents the L-HAND task, the subtraction at the top is the SUBTRACTION task and the shape on the right represents the ROTATION task). One second later, at $\mathrm{t}=3 \mathrm{~s}$ the instruction is provided to the participant in the form a red arrow, displayed for 1.250 s, pointing in the direction of the task to be performed. Finally, once the instruction has been given, the participant is provided with feedback (for $4 \mathrm{~s}$ ) in the shape of a blue feedback bar, the direction of which indicates which task has been recognised and the length of which represents the confidence of the system in the recognition of this task. 
exercises. In order for VC and SA training to be comparable, we proposed 4 different kinds of exercises. The first and second exercises consisted in finding synonyms and antonyms, respectively. The third consisted in completing sentences with analogies and the last one consisted in determining the meaning of a proverb. During each session, participants performed 5 runs, each lasting 7 minutes. At each run a different exercise was presented, but the instructions were always the same: a target word/sentence was presented at the top of the screen, followed by four options ; among these four options, the participants had to select the two correct ones. For example, an exercise on synonyms might provide the user with the word Big, followed by four options : Huge, Edible, Large and Fruitful. The goal of the participant is to select the two correct answers. A 7 minute time limit was set for participants to complete as many items as possible. From the second run onwards, participants were able to click on a check button in order to receive feedback (i.e., to know whether they had answered correctly or not).

\section{Pre-Study $\sharp 1$ : Determining the Degree OF DIFFICULTY OF SA AND VC TRAINING EXERCISES}

The aim of this first pre-study was to determine the degree of difficulty, both objective (i.e. performance) and subjective (i.e. perceived difficulty), of the exercises proposed in the SA and VC training protocols. This analysis enabled us to check experimentally if the different exercises were indeed associated with increasing degrees of difficulty, as recommended by instructional design literature [11]. Also, it enabled us to assess whether the SA and VC training protocols require participants to mobilise the same level of cognitive resources.

\section{A. Participants}

Each participant $(\mathrm{N}=31,9$ women) performed 8 exercises (4 SA and $4 \mathrm{VC}$ exercises). Half ( $\mathrm{N}=16,4$ females) of the participants started with $4 \mathrm{SA}$ questionnaires and finished with the $4 \mathrm{VC}$ questionnaires, while the other half $(\mathrm{N}=15,5$ females) started with $4 \mathrm{VC}$ questionnaires and then completed $4 \mathrm{SA}$ questionnaires. The $\mathrm{SA}$ and $\mathrm{VC}$ exercises were performed in a counterbalanced order across the participants. This study along with all of the following studies were conducted in accordance with the relevant guidelines for ethical research according to the Declaration of Helsinki. All the participants signed an informed consent form at the beginning of all the experiments.

\section{B. Materials and Methods}

As stated earlier, each participant performed 8 on-line exercises (4 SA and $4 \mathrm{VC}$ ). Each exercise comprised 8 items. At the

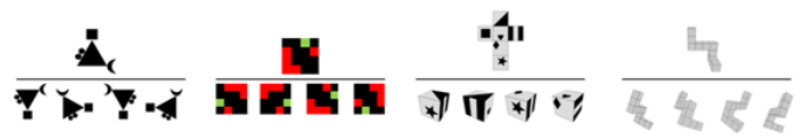

Fig. 2. One item per exercise included in the Spatial Ability training: the shape on top is the target, and the participant must identify the two shapes that are identical to the target among the four below.From the left to the right are displayed the shapes, matrices, cubes, arms exercises. end of each exercise, they completed a Likert-scale in order to rate their perceived effort from 0 to 10 . The statistical analysis enabled us to detect any significant differences between the exercises (and thus between the training protocols), both in terms of score (called "objective difficulty" in the analysis) and perceived effort (called "subjective difficulty" in the analyses).

\section{Results}

We performed four analyses of variance (ANOVA) to assess the differences between the exercises of each training protocol, i.e., SA and VC, both in terms of objective (score) and subjective (perceived effort) difficulty. Concerning the SA training, the results showed a main effect of the exercise both in terms of objective difficulty $\left(\mathrm{D}(30)=102.900, \mathrm{p} \leq 0.001, \eta^{2}=0.774\right)$ and in terms of subjective difficulty $(\mathrm{D}(30)=118.637, \mathrm{p} \leq 0.001$, $\left.\eta^{2}=0.798\right)$. Post-hoc analyses indicated that the shapes exercise was associated with significantly better scores and lower perceived effort than the matrices exercise, itself being easier and requiring less subjective effort than the arms exercise, itself being rated easier and requiring less subjective effort than the cubes exercise. On the other hand, concerning the VC training, the ANOVA revealed a main effect of the exercise in terms of objective difficulty $\left(\mathrm{D}(30)=22.942, \mathrm{p} \leq 0.001, \eta^{2}=0.433\right)$ but not in terms of subjective difficulty $(\mathrm{D}(30)=2.098, \mathrm{p}=0.158$, $\eta^{2}=0.065$ ). Post-hoc analyses revealed that the synonyms and analogies exercises were associated with similar scores while the antonyms exercise required significantly more subjective effort, and the proverbs exercise even more perceived difficulty. Finally, we performed a two 2-way ANOVA for repeated measures in order to compare the two training protocols in terms of score and perceived effort. The first ANOVA revealed no difference in terms of scores between the SA and VC trainings $(p=0.902)$ while the second revealed a main effect of the training type on the perceived effort required to complete the task $(\mathrm{p} \leq 0.001)$ : post-hoc analyses showed that the SA and $\mathrm{VC}$ exercises were equivalent except from the cube exercise that was perceived as much more difficult.

\section{Discussion}

This first pre-study enabled us to to verify that both SA and VC training included exercises with different levels of difficulty, and thus followed the recommendations from instructional design [11]. Participants rated VC and SA exercises as demanding (subjective effort), except from the cube exercise that appeared to be much more demanding (which could be due to the fact that difficult $\mathrm{VC}$ exercises require previous knowledge while difficult SA exercises can be solved by thinking about it). Their scores (objective effort) were also equivalent for both training types, suggesting a comparable degree of difficulty. Since the exercises from the VC and SA training protocols proved to have an equal complexity, we were able to use VC training as a control. The next step was to verify the effectiveness of the SA training protocol for improving spatial abilities. 


\section{Pre-Study $\sharp 2$ : VAlidating the SA AND VC TRAINING PROTOCOLS}

A second pre-study was carried out in order to evaluate the effectiveness of our SA and VC training protocols. Indeed, although we designed the questionnaire exercises with theoretical considerations in mind, we still had to verify whether our SA training protocol actually led to an improvement of the user's spatial abilities. Conversely, we also had to ensure that the $\mathrm{VC}$ training protocol did not improve SA, in order for the control group to be able to use it without affecting the outcome. Accordingly, enrolled two groups of participants who completed the entire SA or VC training protocols. Their spatial abilities were evaluated before and after training in order to assess the impact of each training protocol on SA.

\section{A. Participants}

The participants (N=19, 10 women) first took part in a session during which their SA and other cognitive abilities were measured. They were then divided into two homogeneous groups in terms of gender and mental rotation scores obtained during this first session. The first group ( $\mathrm{N}=9,5$ women) completed the SA training protocol, i.e., they performed each of the three SA training sessions over several days. The second group ( $\mathrm{N}=10,5$ women) completed the $\mathrm{VC}$ training protocol, with sessions being similarly spread out over different days.

\section{B. Materials and Methods}

During the first session, participants performed the mental rotation test [8] which assesses spatial visualisation abilities, i.e., SA. Their training was then performed online and at home, with a maximum of one session per day. They then performed the same psychometric test again in the final session.

\section{Results}

In order to assess the effectiveness of the SA training protocol, we performed a two way ANOVA for repeated measures. In this manner, we were able to detect any significant differences between pre- and post-training mental rotation scores, as a function of both the group (SA vs. VC) and gender (as SA are known to be associated with an important gender effect). However, before performing the ANOVA, it was necessary to check that the participants from both groups had similar SA at the beginning of the experiment, i.e., before training. Results revealed that the variances were equal between the groups $(\mathrm{F}(19)=0.011, \mathrm{p}=0.917)$, as well as the mean rotation scores $(\mathrm{t}(19)=0.402, \mathrm{p}=0.692)$. Then, the ANOVA revealed, as stated in the literature, a main effect of the gender $(\mathrm{D}(1,17)=5.056$, $\left.\mathrm{p} \leq 0.05, \eta^{2}=0.229\right)$. Second, it revealed a rotationScore * group interaction effect $\left(\left(\mathrm{D}(1,17)=7.388, \mathrm{p} \leq 0.05, \eta^{2}=0.303\right)\right.$ : participants in the SA group made significantly greater improvements compared to those in the VC group.

\section{Discussion}

This second pre-study allowed us to confirm that participants performing the SA training protocol tend to improve their spatial abilities significantly better than participants in the $\mathrm{VC}$ training group. Although participants in the $\mathrm{VC}$ group did improve their SA, improvements were only minor and were more likely due to the fact that they had completed each questionnaire twice, and consequently were more familiar with the questionnaire the second time. Nonetheless, the marked improvement in SA abilities in the SA training group does confirm that the training exercises that we designed do indeed lead to improved spatial abilities. It was then possible to integrate this training approach in an MI-BCI training protocol with a view to assessing its impact on BCI performances. This was implemented in the following pilot study.

\section{Pilot Study: Validating the 3 Different BCI TRAINING REGIMES}

Having verified the effectiveness of our SA training protocol, we conducted a first pilot $\mathrm{BCI}$ experiment to validate the 3 BCI training protocols: the standard $\mathrm{BCI}$ training including SA training, the standard BCI training protocol as a control, as used in [7], and a standard BCI training protocol which included VC training tasks, as another control. This pilot study only included 3 subjects, one in each group. As such it cannot lead to any statistically meaningful comparisons between training types. However, it should provide us with relevant and useful insights into each training protocol, before performing a large scale comparison in the future, with multiple participants in each group.

\section{A. Participants}

Each of our 3 participants was assigned to a different condition: two control conditions (standard BCI training and $\mathrm{VC}$ training) and one experimental condition (SA training). The participant in the first control condition (male, 21 years old) took part in 6 standard MI-BCI training sessions (MI condition). This participant is one of the participants from a previous study [7], and his performances were reported in that publication. He was selected for having an average BCI performance and mental rotation score as close as possible to that of the other 2 participants. The participant in the second control condition (male, 21 years old) took part in 3 standard MI-BCI training sessions and $3 \mathrm{VC}$ training sessions (VC condition). Finally, the participant in the experimental condition (male, 25 years old) took part in 3 standard MI-BCI training sessions and $3 \mathrm{SA}$ training sessions (SA condition). All these three participants were males and had at least a degree equivalent to an A-level. They were all right handed and healthy, i.e. they did not suffer from any neurological or psychiatric disorder that could impact their EEG signals or prevent them from focusing on a 2-hour long task.

\section{B. Material and Method}

1) Experimental paradigm: Each participant performed 3 MI-BCI sessions and $3 \mathrm{MI}$, SA or VC training sessions: they all started with one MI-BCI session after which they completed their training (MI, SA or VC) according to the condition they had been attributed to, and finished with two MI-BCI sessions.

All the sessions were spread out over up to 2 weeks, to ensure that each participant never did more than 1 training session 
per day. At the end of the first session and at the beginning of the fifth session, i.e. before and after the condition-specific training, all participants completed the Mental Rotation Test (around 10 minutes) to verify whether their training had improve their Spatial Abilities.

EEG cap installation lasted around 20min. Naturally, EEG was measured during the MI-BCI training sessions, but also during the first and last SA/VC training sessions (i.e., sessions 2 and 4) in order to detect any possible neural correlates of SA/VC training. Participants then completed five 7-minute runs during which they had to perform the required task (MI, SA or VC), for a total duration of approximately 60 minutes (including breaks between the runs). At the end of each session, participants were debriefed (10 minutes).

2) EEG recording and machine learning: The EEG signals were recorded from a g.USBamp amplifier (g.tec, Graz, Austria), using 30 scalp electrodes (F3, Fz, F4, FT7,FC5, FC3, FCz, FC4, FC6, FT8, C5, C3, C1, Cz, C2, C4, C6, CP3, CPz, CP4, P5, P3, P1, Pz, P2, P4, P6, PO7, PO8, 10-20 system), sampled at $256 \mathrm{~Hz}$, referenced to the left ear and grounded to $\mathrm{AFz}$, as in [7]. In order to classify the 3 mental imagery tasks on which our BCI is based, first, EEG signals were bandpass filtered in $8-30 \mathrm{~Hz}$, using a Butterworth filter of order 4. Then EEG signals were spatially filtered using 3 sets of Common Spatial Pattern (CSP) filters [12]. The CSP algorithm aims at finding spatial filters whose resulting EEG band power is maximally different between two classes. Each set of CSP filters was optimised on the calibration run of each user (i.e., the first run of the first session) to discriminate EEG signals for a given class from those for the other two classes. We optimised 2 pairs of spatial filters for each class, corresponding to the 2 largest and lowest eigen values of the CSP optimisation problem for that class, thus leading to 12 CSP filters. The band power of the spatially filtered EEG signals was then computed by squaring the signals, averaging them over the last 1 second time window (with 15/16s overlap between consecutive time windows) and log-transformed. These resulted in 12 bandpower features that were fed to a multi-class shrinkage Linear Discriminant Analysis (sLDA) [13], built by combining three sLDA in a one-versus-the-rest scheme. As for the CSP filters, the sLDA were optimised on the EEG signals collected during the calibration run, i.e., during the first run of the first session. The resulting classifier was then used online to differentiate between left-hand motor imagery, mental rotation and mental subtraction during the subsequent MI-BCI runs. The sLDA classifier output (i.e., the distance of the feature vector from the LDA separating hyperplane) for the mental imagery task to be performed was used as feedback provided to the user. In particular, if the required mental task was performed correctly (i.e., correctly classified), a blue bar with a length proportional to the LDA output and extending towards the required task picture was displayed on screen and updated continuously. If the required mental task was not correctly classified, no feedback was provided, as in [7]. To reduce between session variability, the LDA classifiers biases were re-calculated after the first run of the sessions 5 and 6 , based on the data from this first run, as in [7]. EEG signals were recorded, processed and visually inspected with OpenViBE [14].

\section{Preliminary results}

We measured the MI-BCI performances for each run of the 3 MI-BCI sessions common to all participants, (sessions 1, 5 and 6). Performances were measured in two ways: as the mean classification accuracy over the whole feedback period (see Figure 3), or as the peak classification accuracy, i.e., as the accuracy for the best time window of each run (see Figure 4). Overall, the participant in the MI-BCI condition reached an average accuracy of $46.9 \%$ for the 3 classes, the participant in the VC condition $53.8 \%$ and the one in the SA condition $41.5 \%$. Their mental rotation scores were respectively 25,34 (35 after $\mathrm{VC}$ training) and 12 (32 after SA training). Based on the model we proposed in [7] that enables performances to be predicted based on the participant's learning style, tension, autonomy and abstractedness, the predicted MI-BCI accuracies were $46.1 \%, 54.4 \%$ and $52.4 \%$. Thus, for both the participants having average/good mental rotation scores (the ones in the MI and VC conditions), the model predicted their performance with an error rate of $0.8 \%$ and $0.5 \%$. However, as was the case in [7], the model over-estimated the performance of the participant with low mental rotation scores. It is likely to be due to the strong correlation between SA and BCI performance: despite the participant has the profile to be a good BCI performer based on the model, SA's weight on BCI performance is bigger and thus conditions the performance.

\section{Discussion}

In terms of MI-BCI performance, it can be observed that both the participant from the MI-BCI condition and the one who was attributed to the SA condition, improved their peak accuracy over certain runs. The participant from the VC condition improved his peak substantially and his mean accuracy over runs and sessions. While such results with only 1 participant per condition cannot lead to any significant comparison between the training approaches, it still provides some interesting insights. First, it seems to confirm once more the impact of initial SA on MI-BCI performances. Indeed, the participant from the VC condition had the highest SA before and after training, that is to say a high mental rotation score,

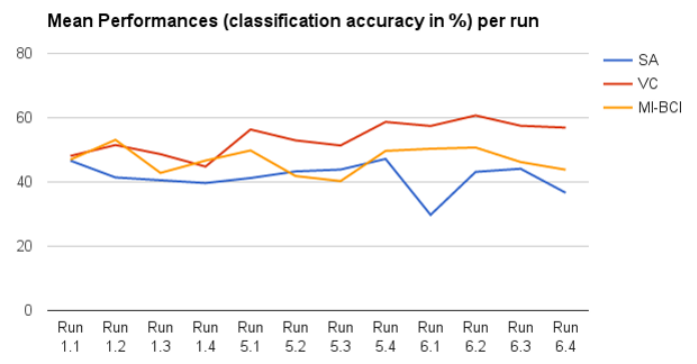

Fig. 3. Mean BCI performance (percentage of correct classification) for each run and each participant in the pilot study. 
above the male average [8]. Indeed his score was 34 before and 35 after the VC training, and this participant achieved the best BCI performance and progression. The participant who was attributed to the SA condition had the lowest mental rotation score, much lower than the male average: 12 before training. He nevertheless managed to reach BCI performances similar to those of the control participant (MI-BCI condition) while having practiced actual BCI control for half as long (3 sessions versus 6), and having a lower initial SA. These results suggest that integrating SA training sessions in an MI-BCI training protocol is feasible and thus worth being further explored in a full scale study with multiple participants. Second, the performance of these participants consolidates the validity of the model proposed in [7] as it enabled accurate performance prediction for both the participants having normal/good SA. However, as was the case in [7], it overestimated the performance of the participant having low mental rotation scores. This result reinforces the hypothesis that certain personality traits of the user (tension, self-reliance, abstractedness and active learning style) have an impact on MI-BCI performance, and stresses the predominant effect of spatial abilities.

\section{Global Discussion}

In this paper we designed and validated an SA training approach and incorporated it into an MI-BCI training protocol. Indeed, SA have been shown to be correlated to MI-BCI performances, which led us to the idea of training BCI users' SA with the hope of improving their MI-BCI performances. We thus designed an SA training protocol, and characterised the difficulty of the different training exercises in order to ensure that the training protocols comprised exercises with various degrees of difficulty, as recommended according to instructional design. We then used $\mathrm{N}=19$ subjects to verify that the SA training protocol did indeed lead to a significant improvement in SA, this improvement being significantly greater than that obtained after completing a control training protocol based on VC exercises. Finally, we performed a pilot study with 3 participants in which this SA training approach was integrated within a standard MI-BCI training protocol. Results of this pilot study seemed to confirm the impact of initial SA on MI-BCI control performances, and that the integration of SA training into standard BCI training is feasible. Although very

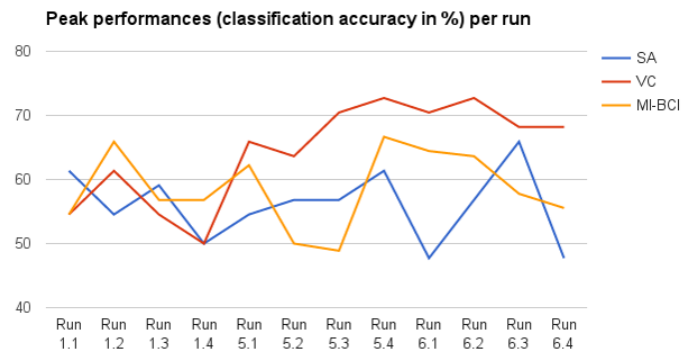

Fig. 4. Peak BCI performance (percentage of correct classification) for each run and each participant in the pilot study. preliminary, and without any statistical significance, which currently prevents us from drawing any strong conclusion, such results encourage further investigation. Indeed, they suggest that an MI-BCI training procedure might not only include MIBCI tasks but also other carefully designed cognitive training tasks. An interesting parallel can be made here with training for sports performance. Indeed, an athlete training for martial arts for instance will not only practice the martial art. His training regime will also incorporate running, stretching, weighttraining, developing mental focus, etc. Since BCI control is also a skill that can be learned, it would make sense that the inclusion of specific cognitive training contributes towards improving MI-BCI performance. We will explore that line of research in the near future and attempt to validate the present results on multiple subjects in each of the three conditions. We will also study the neural correlates of SA training in more detail, to try to acquire a deeper understanding of the possible impact of such training on MI-BCI control performances. Overall we aimed at providing a new training task for BCI user training, thus enriching the currently poor repertoire of exercises that are available for our BCI users.

\section{ACKNOWLEDGMENT}

This work was supported by the French National Research Agency with project REBEL (grant ANR-15-CE23-0013-01) and IdEx Bordeaux.

\section{REFERENCES}

[1] J. Wolpaw and E. Wolpaw, "BCI: Principles and practice," 2012.

[2] K. K. Ang and C. Guan, "Brain-computer interface for neurorehabilitation of upper limb after stroke," Proc. IEEE, vol. 103, no. 6, 2015.

[3] B. Z. Allison and C. Neuper, "Could anyone use a BCI?" in Braincomputer interfaces. Springer, 2010, pp. 35-54.

[4] C. Neuper and G. Pfurtscheller, "Neurofeedback training for BCI control," in Brain-Computer Interfaces. Springer, 2009, pp. 65-78.

[5] J. R. Wolpaw, N. Birbaumer, D. J. McFarland, G. Pfurtscheller, and T. M. Vaughan, "Brain-computer interfaces for communication and control," Clinical neurophysiology, vol. 113, no. 6, pp. 767-791, 2002.

[6] F. Lotte and C. Jeunet, "Towards improved bci based on human learning principles," in 3rd Int Winter Conf BCI. IEEE, 2015, pp. 1-4.

[7] C. Jeunet, B. N'Kaoua, S. SUbramanian, M. Hachet, and F. Lotte, "Predicting mental imagery-based BCI performance from personality, cognitive profile and neurophysiological patterns," PLOS ONE, vol. 10, no. 12, p. e0143962, 2015.

[8] S. G. Vandenberg and A. R. Kuse, "Mental rotations, a group test of three-dimensional spatial visualization," Perceptual and motor skills, vol. 47, no. 2, pp. 599-604, 1978.

[9] C. Jeunet, E. Jahanpour, and F. Lotte, "Why standard brain-computer interface (BCI) training protocols should be changed: An experimental study," Journal of Neural Engineering, 2016.

[10] E. V. Friedrich, C. Neuper, and R. Scherer, "Whatever works: A systematic user-centered training protocol to optimize brain-computer interfacing individually," PloS one, vol. 8, no. 9, p. e76214, 2013.

[11] F. Lotte, F. Larrue, and C. Mühl, "Flaws in current human training protocols for spontaneous brain-computer interfaces: lessons learned from instructional design," Frontiers in Human Neurosciences, 2013.

[12] H. Ramoser, J. Muller-Gerking, and G. Pfurtscheller, "Optimal spatial filtering of single trial EEG during imagined hand movement," IEEE Trans Rehab Eng, vol. 8, no. 4, pp. 441-446, 2000.

[13] F. Lotte and C. Guan, "Learning from other subjects helps reducing brain-computer interface calibration time," in Proc. ICASSP, 2010.

[14] Y. Renard, F. Lotte, G. Gibert, M. Congedo, E. Maby, V. Delannoy, O. Bertrand, and A. Lécuyer, "OpenViBE: an open-source software platform to design, test, and use brain-computer interfaces in real and virtual environments," Presence: teleoperators and virtual environments, vol. 19 , no. 1 , pp. 35-53, 2010. 